\title{
Aspectos de usabilidade de mobile learning voltado para usuários com restrições decorrentes da idade
}

\author{
Rommel Vieira Carneiro ${ }^{1}$ \\ Lucila Ishitani ${ }^{1}$
}

\begin{abstract}
Resumo: A tendência de envelhecimento da população mundial provocou um crescimento no número de pessoas na faixa de idade acima de 60 anos, também conhecida por terceira idade. Essas pessoas necessitam atualizar seus conhecimentos na tentativa de se manterem ativas e inseridas na sociedade. Nesse contexto, a educação a distância (EaD) apresenta-se como uma alternativa interessante, oferecendo conteúdo por meio da internet, com flexibilidade de hora e local de uso. Os dispositivos móveis facilitam o acesso a recursos de aprendizagem, todavia, os aplicativos requerem adaptações para as pessoas da terceira idade que, em geral, possuem uma ou mais restrições decorrentes da idade. Com o objetivo de avaliar a aderência dessas tecnologias ao grupo de pessoas da terceira idade, foram realizados testes de usabilidade com esse público na utilização de dispositivos móveis com um material previamente preparado. Foram identificados alguns aspectos relevantes para o público da terceira idade, bem como recomendações de melhorias no desenvolvimento de conteúdo. Foi também percebida grande aderência dos dispositivos selecionados ao público da terceira idade, sendo 9 dos 11 participantes os que manifestaram interesse no uso dessas tecnologias.
\end{abstract}

Palavras-chave: Mobile learning. Terceira idade. Usabilidade.

\begin{abstract}
The global trend of population aging has led a growth in the number of people over 60 years. These people need to update their knowledge and capabilities to remain active and inserted in the society. In this context, e-learning is an interesting alternative, offering relevant content through the Internet, anywhere and anytime. Mobile devices provide access to learning resources, however, they require adjustments to bring all these technological advances to the older people, who, in general, has one or more restrictions due to ageing. Aiming to evaluate the adherence of these technologies to the seniors, usability tests were conducted with this audience based on the use of mobile devices with a previously prepared material. It was identified relevant usability aspects to this public as well as recommendations for improvements in content development. Results pointed that 9 people among 11 interviewed manifested great interest in using these technologies.
\end{abstract}

Keywords: Elderly. Mobile learning. Usability.

\section{Introdução}

Segundo estatísticas do último censo do Instituto Brasileiro de Geografia e Estatística (IBGE) [1], a proporção da população brasileira acima de 60 anos cresceu de 8,60\% em 2002 para 11,00\% em 2010. Essa tendência de envelhecimento da população é global. Por isso, cada vez mais indivíduos acima de 60 anos necessitarão atualizar seus conhecimentos e adquirir novas competências para se manterem ativos e inseridos no contexto social.

Paralelamente a essas mudanças, as tecnologias embarcadas nos dispositivos móveis vêm ganhando espaço e mudando o cenário dos meios de comunicação e, consequentemente, de áreas como a educação. A partir dos dispositivos móveis, novas áreas de estudo como a aprendizagem móvel ou mobile learning (m-learning) ganharam relevância e percebe-se o surgimento de um novo ambiente com potencial de aplicação em diversas abordagens pedagógicas [2].

${ }^{1}$ Mestrado em Informática, PUC Minas - São Gabriel - Belo Horizonte (MG) - Brasil

\{rommelcarneiro@gmail.com, lucila@pucminas.br\}

http://dx.doi.org/10.5335/rbca.2014.3426

Revista Brasileira de Computação Aplicada (ISSN 2176-6649), Passo Fundo, v. 6, n. 1, p. 81-94, abr. 2014 
Os dispositivos móveis oferecem grande liberdade de uso e também grandes desafios para a adoção do mobile learning: o tamanho reduzido das telas, a dificuldade na entrada e saída de informação, a baixa capacidade de processamento e memória, a baixa autonomia de baterias, o alto custo dos equipamentos e da conectividade e a diversidade de plataformas [2,3]. Além disso, à medida que a idade avança, as pessoas começam a enfrentar alterações nas suas capacidades cognitivas e motoras. Isso exige que os recursos tecnológicos utilizados no processo de ensino-aprendizagem sejam adaptados para lhes permitir o acesso e o aproveitamento de forma plena.

O objetivo deste trabalho é apresentar os resultados de uma avaliação de adequação dos dispositivos móveis em iniciativas de educação e informação junto às pessoas da terceira idade e propor recomendações de usabilidade na produção de conteúdo voltado para esse público. No intuito de atingir esses resultados, foi realizado um levantamento e uma organização das melhores práticas no desenvolvimento de conteúdo educacional para o público da terceira idade.

O material de teste foi desenvolvido a partir de conteúdos parcialmente extraídos da internet. A adaptação do conteúdo foi realizada inicialmente com base nas melhores práticas levantadas e posteriormente com um préteste conduzido com pessoas pertencentes ao grupo da terceira idade.

A validação de aderência se deu por meio de testes de usabilidade. Após a experiência realizada com 11 idosos, apesar de oito dos participantes nunca terem utilizado dispositivos móveis como os que foram empregados no teste, nove manifestaram interesse no uso dessas tecnologias e 10 consideraram os equipamentos altamente adequados para a prática de $\mathrm{EaD}$, confirmando que esses dispositivos poderiam substituir os mecanismos utilizados até então.

Nas próximas seções, serão detalhados os principais conceitos relacionados ao tema deste artigo, a metodologia utilizada, os testes realizados, os resultados obtidos com a pesquisa e as conclusões a que se chegou.

\section{Aprendizagem na terceira idade}

De acordo com Machado [7], públicos de faixas etárias distintas aprendem de formas diferenciadas. Assim, a aprendizagem de adultos e idosos requer uma abordagem diferenciada em relação ao aprendizado de crianças e adolescentes. Em função do conhecimento prévio e das experiências de vida, é importante associar o aprendizado à realidade do aprendiz de maneira que o conhecimento adquirido possa ser aplicado de forma prática e imediata.

Existem pesquisas que buscam traçar teorias e modelos que possam orientar o aprendizado de adultos e idosos. Merriam, Caffarella e Baugartner [8] apontam a andragogia como o melhor modelo para o aprendizado de adultos, embora outros modelos estudados ofereçam contribuições relevantes. Os autores fundamentam a andragogia com base em cinco suposições sobre os aprendizes adultos:

- à medida que um indivíduo amadurece, deixa de ter uma personalidade dependente e passa a se direcionar de forma autônoma;

- um adulto acumula uma experiência crescente, o que constitui um recurso rico para o aprendizado;

- a prontidão de um adulto pelo aprendizado está diretamente relacionada às tarefas de desenvolvimento do seu papel social;

- os adultos são direcionados pela aplicação imediata do conhecimento na prática. Com isso privilegiam a abordagem voltada para a resolução de problemas;

- os adultos são motivados a aprender preferencialmente por fatores internos que por fatores externos. A satisfação em aprender e o alcance de metas pessoais são exemplos que têm maior efeito frente a recompensas e incentivos externos.

No caso de idosos, deve-se considerar, ainda, as restrições decorrentes da idade, tais como ([9, 21]):

- declínio da percepção visual e da audição;

Revista Brasileira de Computação Aplicada (ISSN 2176-6649), Passo Fundo, v. 6, n. 1, p. 81-94, abr. 2014 
- declínio da capacidade de memorização e da velocidade de processamento de novas informações;

- redução do controle motor e da destreza.

O reconhecimento dessas características em aprendizes idosos é fundamental, pois, somente tratando de forma apropriada as necessidades específicas do público-alvo é possível garantir a correta adequação da interface do sistema com o usuário e a eficácia do ambiente que se espera construir.

\section{Acessibilidade e usabilidade}

Nos domínios das tecnologias da informação, a acessibilidade é uma condição para prover o acesso universal e, nesse sentido, pode ter diversas interpretações [10]:

1. em alguns meios, pode se referir à conectividade;

2. em termos econômicos, está associada à infraestrutura de acesso;

3. relacionada aos meios cognitivos e à educação, refere-se à disponibilidade da informação ou ainda à usabilidade dos recursos por pessoas com necessidades especiais.

O World Wide Web Consortium (W3C), uma organização voltada para a criação de padrões abertos para a Web, possui iniciativas específicas no sentido de orientar o processo de desenvolvimento de conteúdo para dispositivos móveis. Tais iniciativas buscam a melhoria da acessibilidade e usabilidade do conteúdo elaborado [11]. Na recomendação do W3C intitulada Web Content Accessibility Guidelines (WCAG), [12] destacam-se quatro princípios da acessibilidade sobre os quais um conteúdo deve ser criado e apresentado: perceptível, operável, compreensível e robusto. As recomendações publicadas pelo W3C são direcionadas para o processo de desenvolvimento de conteúdo no contexto da Web. Apesar disso, uma vez consideradas as particularidades das tecnologias envolvidas em mobile learning, uma grande parte dessas recomendações aplica-se de maneira similar. Portanto, as recomendações do W3C são de grande valia para este estudo.

$\mathrm{O}$ termo usabilidade refere-se à medida na qual um produto ou serviço pode ser usado por usuários determinados para alcançar objetivos específicos com eficácia, eficiência e satisfação em um contexto específico de uso [13]. A norma NBR 9241-11 apresenta essas dimensões fundamentais de usabilidade da seguinte maneira:

- eficácia - atendimento aos objetivos especificados;

- eficiência - recursos empregados frente à abrangência dos resultados obtidos;

- satisfação - ausência de desconforto no uso do produto ou serviço.

Preece, Rogers e Sharp [14] identificam um conjunto mais abrangente de dimensões de usabilidade no projeto de um sistema interativo, às quais denominam metas de usabilidade. São elas: ter eficácia no uso; ter eficiência no uso; oferecer segurança; ter boa utilidade; ser fácil no aprendizado; ser fácil de lembrar a forma de uso.

Os métodos tradicionais para testes de usabilidade podem requerer adequação para se conseguir resultados efetivos com um público de pessoas idosas [15]. No estudo realizado por Nahm e outros [15], foram avaliados websites de saúde voltados para esse tipo de público. Conforme os autores, a intervenção do pesquisador, durante os testes, muitas vezes auxilia no processo de obtenção dos resultados, embora a recomendação da literatura seja contrária a essa prática. Tal constatação deve-se à pequena experiência dos participantes no uso da internet, o desconhecimento prévio do funcionamento dos sites e algumas características inerentes ao perfil das pessoas idosas, como a dificuldade de se lembrar de ocorrências passadas durante os testes para consolidação posterior. Ainda segundo os autores, embora diversos especialistas preconizem o uso de métricas quantificáveis nos testes de usabilidade, tais como tempo para conclusão de uma tarefa e frequência de pedidos de ajuda, a avaliação desses atributos torna-se problemática em função da falta de testes com esse público específico. 


\subsection{Usabilidade para a terceira idade}

Existem diversos estudos que buscam identificar e estruturar um conjunto de melhores práticas para a elaboração de conteúdo web voltado para a terceira idade $([17,18,19,20])$. Os resultados encontrados nesses estudos são igualmente válidos no contexto de mobile learning, uma vez que a web é um dos principais meios de acesso ao conteúdo educacional por meio de dispositivos móveis. Dentre esses trabalhos, cabe destacar o do National Institute on Aging (NIA), que disponibiliza artigos a respeito da usabilidade de sites web voltados para idosos, além de um vasto material sobre a saúde, já adaptado para atender as recomendações apresentadas. Parte do material disponibilizado pelo NIA foi utilizado na avaliação realizada neste trabalho. As recomendações encontradas, cuja compilação é apresentada ao final desta seção, abrangem a estruturação do conteúdo, o design dos sites, a preocupação com os mecanismos de navegação pelo conteúdo e a inclusão de mecanismos de apoio à utilização do site.

Utilizando as recomendações geradas pelo NIA, Becker [22] realizou um estudo de usabilidade por meio da avaliação de 125 sites web com conteúdos sobre saúde. A autora concluiu que a maioria dos sites avaliados não é amigável para o público da terceira idade, apontando como os maiores problemas encontrados: a falta de tratamento do tamanho das letras, a necessidade de destreza com o mouse para utilização dos menus de opções e a ausência de páginas de ajuda e mapas do conteúdo. Além dos aspectos relacionados ao projeto dos sites, a pesquisadora ressalta que o tempo médio gasto na carga e exibição do conteúdo é duas vezes maior que o tempo aceitável, recorrendo ao citado por Nielsen e Nahir, que recomendam um tempo máximo de dez segundos.

Foram encontrados poucos trabalhos voltados para a avaliação da usabilidade de dispositivos móveis com o público da terceira idade. Gonçalves, Neris e Ueyama [23] apresentam um estudo sobre o design de interfaces flexíveis em celulares para o público de terceira idade. Mol e Ishitani [24] apresentam uma avaliação da interface de um jogo educativo em celular para o público da terceira idade com o objetivo de validar se as métricas de usabilidade recomendadas para computadores são igualmente válidas para celulares, considerando o público em questão. Strengers [25] realizou uma pesquisa que confirmou que os requisitos para interfaces de smartphones são semelhantes aos definidos para sistemas a serem utilizados em desktops, com poucas exceções, tais como: evitar o uso de teclado na tela de smartphones, evitar animações e limitar o número de opções a serem mostradas, para evitar confusão e ter espaço para mostrar botões e ícones em tamanho visível. Por sua vez, Leitão e Silva [21] desenvolveram um estudo específico sobre o tamanho ideal e o espaçamento entre objetos a serem selecionados. Os resultados da pesquisa sugerem tamanhos para objetos, mas não identificam efeitos significativos relacionados a espaço entre objetos.

A escassez de trabalhos nessa linha de pesquisa e um aumento no número de pesquisas nos últimos anos demonstram que há muito espaço para a pesquisa de alternativas para o desenvolvimento de material de mobile learning adequado ao público da terceira idade.

A seguir, é apresentada uma compilação de boas práticas a serem adotadas no desenvolvimento de conteúdo de mobile learning para o público da terceira idade. Essa relação é resultado da análise crítica das técnicas e recomendações encontradas nos trabalhos citados nesta seção. O conjunto de orientações é direcionado para o desenvolvimento de conteúdo web, muito utilizado nos dispositivos móveis, mas se aplica também, quase na sua totalidade, a outras plataformas.

As recomendações quanto ao conteúdo são:

- apresentar o conteúdo em seções menores, minimizando tempo de carga e o uso de barras de rolagem;

- reduzir a quantidade de texto para ser lido na tela;

- estruturar o conteúdo em poucos níveis hierárquicos;

- utilizar uma linguagem simples e minimizar o uso de jargões e termos técnicos.

As recomendações quanto ao design do site são:

- utilizar padrões de disposição das informações (templates) de maneira consistente por todo o conteúdo, colocando os mesmos elementos (títulos e subtítulos, menus, botões de navegação) sempre no mesmo local em todas as páginas, com o mesmo formato e tipo de letra;

Revista Brasileira de Computação Aplicada (ISSN 2176-6649), Passo Fundo, v. 6, n. 1, p. 81-94, abr. 2014 
- dispor a informação mais importante no topo;

- utilizar fontes sem serifas, como helvetica e arial;

- utilizar tamanhos de letra entre 12 e 14 pontos para o corpo do texto e entre 18 e 24 para títulos e cabeçalhos;

- utilizar maior espaçamento entre as linhas e os caracteres;

- utilizar o texto justificado à esquerda;

- utilizar tamanhos de linha entre 45 e 60 caracteres, evitando a necessidade de deslocar muito o olho durante a leitura;

- deixar espaço em torno de objetos selecionáveis de forma que não seja necessária muita destreza para selecioná-los;

- utilizar combinações de cores de alto contraste como, por exemplo, letras pretas sobre um fundo branco;

- evitar animações e textos piscando que possam ser fontes de distração;

- evitar fundos de tela com padrões.

As recomendações quanto aos mecanismos de navegação são:

- ser consistente nos mecanismos de navegação, mantendo o mesmo padrão por todo o conteúdo;

- prover instruções claras e numerar cada passo;

- prover informação sobre a localização da página corrente;

- distinguir visualmente os links já acessados daqueles ainda não visitados;

- utilizar clique simples de mouse, evitando clique duplo;

- fornecer ao usuário a visualização de uma única tela por vez e evitar o uso de caixas de diálogo sobrepostas;

- evitar uso de menus de opções cuja abertura e utilização exijam a movimentação do mouse e consequente destreza do usuário.

As recomendações de apoio à utilização e acessibilidade são:

- disponibilizar opção para aumentar o tamanho da letra utilizada nos textos;

- disponibilizar opção para aumentar o contraste da tela;

- prover função para ouvir o texto lido em voz alta;

- prover versões alternativas em texto para o conteúdo multimídia (vídeo e imagens);

- escolher um mecanismo de busca que utilize palavras-chave e não exija símbolos especiais ou conhecimento de termos booleanos.

\section{Metodologia}

\subsection{Participantes}

De acordo com Jakob Nielsen, citado por Bevan [26], existe um número mágico de cinco usuários a serem utilizados em um teste de usabilidade. Com esse número, é possível obter resultados muito similares em casos nos quais os resultados têm o propósito de diagnóstico e obtenção de percepções. Por outro lado, Faulkner [27] afirma que é necessário que o conjunto de usuários seja representativo. Seguindo essa linha, estabeleceu-se como meta um número mínimo de 10 pessoas, dobro do sugerido, para realização dos testes de usabilidade. As pessoas envolvidas nos testes possuíam no mínimo 60 anos. 


\subsection{Testes realizados}

Os testes foram realizados em estudos de campo no ambiente natural das pessoas envolvidas. Essa configuração do teste foi proposital, pois, conforme discutido anteriormente, os aspectos relacionados à mobilidade são de grande importância em iniciativas de mobile learning e, nesse sentido, o contexto é parte integrante na análise dos resultados da aprendizagem por parte dos usuários.

Os testes foram conduzidos em duas etapas:

- pré-teste - contou com um grupo reduzido de pessoas, integrantes do público-alvo e utilizou uma versão inicial do material criado com base nas melhores práticas levantadas e discutidas na fase de levantamento bibliográfico do trabalho. Essa etapa teve por objetivo refinar o material criado e validar o questionário e as informações coletadas no teste de usabilidade;

- teste de usabilidade - realizado com um grupo maior, levou em consideração uma versão do material inicial com as alterações decorrentes das observações feitas durante o pré-teste. O teste de usabilidade constituiuse do preenchimento de um questionário sobre o perfil dos participantes, da observação da utilização do material elaborado e, na sequência, de uma entrevista sobre a experiência dos participantes durante os testes e a opinião deles sobre a utilização dos dispositivos móveis na vida cotidiana.

A avaliação foi estruturada de modo que as informações coletadas fossem tabuladas por meio de intervalos discretos. Isso teve por objetivo otimizar a consolidação dos resultados obtidos e posterior análise.

\subsection{Material de teste}

A expressão "material de teste", mencionada neste trabalho, compreende os dispositivos móveis além do conteúdo educacional e informativo elaborado.

Os dispositivos móveis utilizados foram: telefones inteligentes com interface de tela sensível ao toque entre 3 e 4 polegadas e tablets com tela acima de 10 polegadas. Embora a interface e o sistema operacional dos tipos de dispositivos utilizados sejam similares, optou-se por utilizá-los distintamente, uma vez que a diferença no tamanho das telas representa um aspecto de grande relevância, dadas as características do público-alvo deste trabalho.

Para o desenvolvimento do material utilizado nos testes, foram selecionados conteúdos a partir de sites da internet, adaptados para os dispositivos móveis. Para cada um dos temas, foi elaborado um minicurso sobre o assunto, contendo informações em formatos diversos: texto, imagem e vídeo. Um fator considerado na escolha dos temas foi o da utilidade, uma vez que, segundo o modelo andragógico, os adultos e idosos buscam a aplicação prática daquilo no qual investem tempo para o aprendizado. Foram escolhidos dois temas específicos:

1. "saúde e equilíbrio" - o minicurso preparado sobre o assunto de saúde explora as opções de exercícios físicos que previnem a ocorrência de acidentes e promovem a qualidade de vida. O material utilizado foi extraído do site da web do NIA (nihseniorhealth.gov/exerciseforolderadults), traduzido para o português e adaptado para os dispositivos móveis. A Figura 1 mostra a tela inicial desse material.

2. redes sociais - o material desenvolvido teve parte do seu conteúdo extraído do site Ferramentas Blog (www.ferramentasblog.com) e outra parte desenvolvida no contexto deste trabalho. O conteúdo traz os conceitos das principais redes sociais e orientações sobre a forma de utilização. A Figura 2 mostra a tela inicial desse material.

O material desenvolvido provê funcionalidades para alteração do tamanho do texto utilizado no material, conforme mostrado em destaque na Figura 4.3.

A plataforma utilizada no desenvolvimento do conteúdo dos minicursos foi a estrutura de site web com o padrão HTML5. Foi utilizada ainda uma biblioteca baseada no padrão HTML5, que acelera o processo de desenvolvimento de conteúdo voltado para dispositivos móveis, o jQuery Mobile (jquerymobile.com/). Essa biblioteca, assim como o padrão HTML5, possui compatibilidade com a maioria das plataformas de dispositivos móveis. 
Figura 1: Minicurso sobre saúde

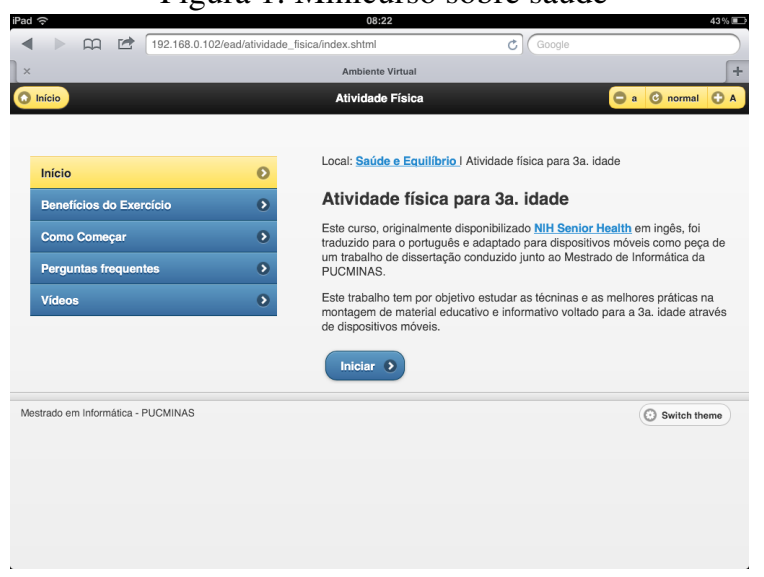

Figura 2: Minicurso sobre redes sociais
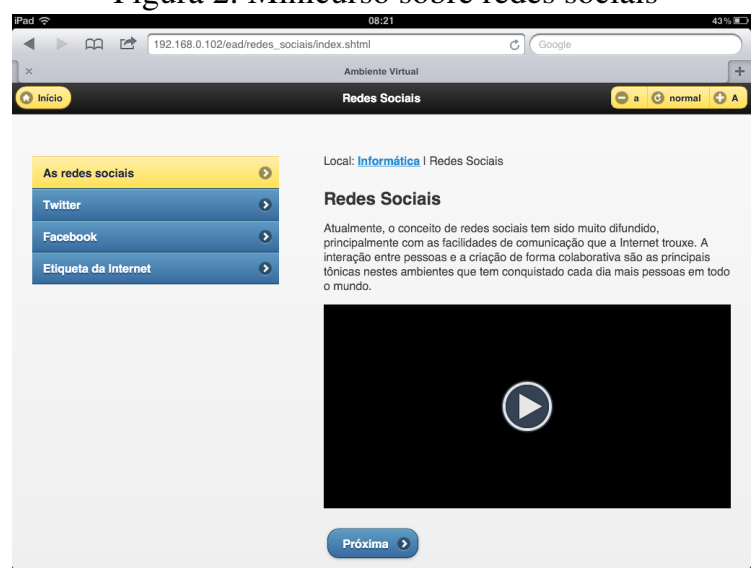

Figura 3: Controle para alteração do tamanho do texto

\section{a (c) normal \& A}

\subsection{Informações coletadas}

As informações coletadas durante o teste, por meio de questionário, são descritas a seguir:

1. Perfil do participante:

- faixa etária do participante do teste. Opções: 60 a 64 anos, 65 a 69 anos, 70 a 74 anos, 75 a 79 anos, 80 a 85 anos, acima de 85 anos;

- sexo do participante do teste. Opções: masculino, feminino;

- meios habituais utilizados pelo participante para o propósito educacional e informativo. Opções: televisão, livros e revistas, jornais impressos, internet, outro.

2. Experiência prévia do participante com tecnologias de informação:

- perfil do participante em termos de utilização de tecnologias. Opções: Grupo A (uso profissional), Grupo B (uso frequente), Grupo C (uso esporádico);

- posse de dispositivo móvel. Opções: sim, não;

- modelo do dispositivo móvel que possui. Opções: celular, smartphone, tablet, iPod ou Nintendo DS ou PSP, PDA ou palmtop, netbook ou notebook ou laptop;

- uso de computador. Opções: sim, não;

- uso de internet. Opções: sim, não.

3. Experiência/percepção do participante com relação ao teste com os equipamentos:

- tamanho da letra do texto adequado. Opções: sim, não, sim (c/ óculos);

- interesse do participante em trocar os meios habituais (livros, revistas, televisão, jornais impressos) pelos dispositivos móveis. Opções: sim, não, talvez;

- tipo de dispositivo preferido, dentre os dispositivos móveis utilizados nos testes. Opções: smartphone, tablet;

- adequação dos dispositivos móveis com o propósito educacional ou informativo, se comparado aos meios habituais. Opções: inadequado, pouco adequado, indiferente, muito adequado;

- interesse em utilizar os dispositivos móveis com o objetivo de aprendizagem. Opções: baixo, médio, alto. 


\section{Resultados do pré-teste}

O pré-teste foi realizado por duas pessoas, uma do sexo masculino, com 61 anos de idade, e a outra pessoa do sexo feminino, com 62 anos de idade. As principais considerações levantadas durante o pré-teste e as ações de adequação realizadas para o teste de usabilidade foram:

- Inicialmente, os participantes demonstraram insegurança no manuseio dos equipamentos utilizados, sendo apontada uma preocupação com relação à fragilidade desses dispositivos. Após algumas explicações sobre sua robustez física e sobre o sistema operacional, os testes transcorreram com maior naturalidade.

Ação de adequação: Foi incorporada uma explicação verbal, de aproximadamente três minutos, sobre a robustez física dos dispositivos móveis na apresentação realizada antes do início do teste de usabilidade. Por meio de uma rápida demonstração de uso, o pesquisador exemplificava o uso da tela sensível ao toque, mostrando sua resistência e ressaltando que o conteúdo não seria alterado, ou possivelmente danificado, durante o teste, por consequência do uso.

Figura 4: Navegação no tablet - antes e depois

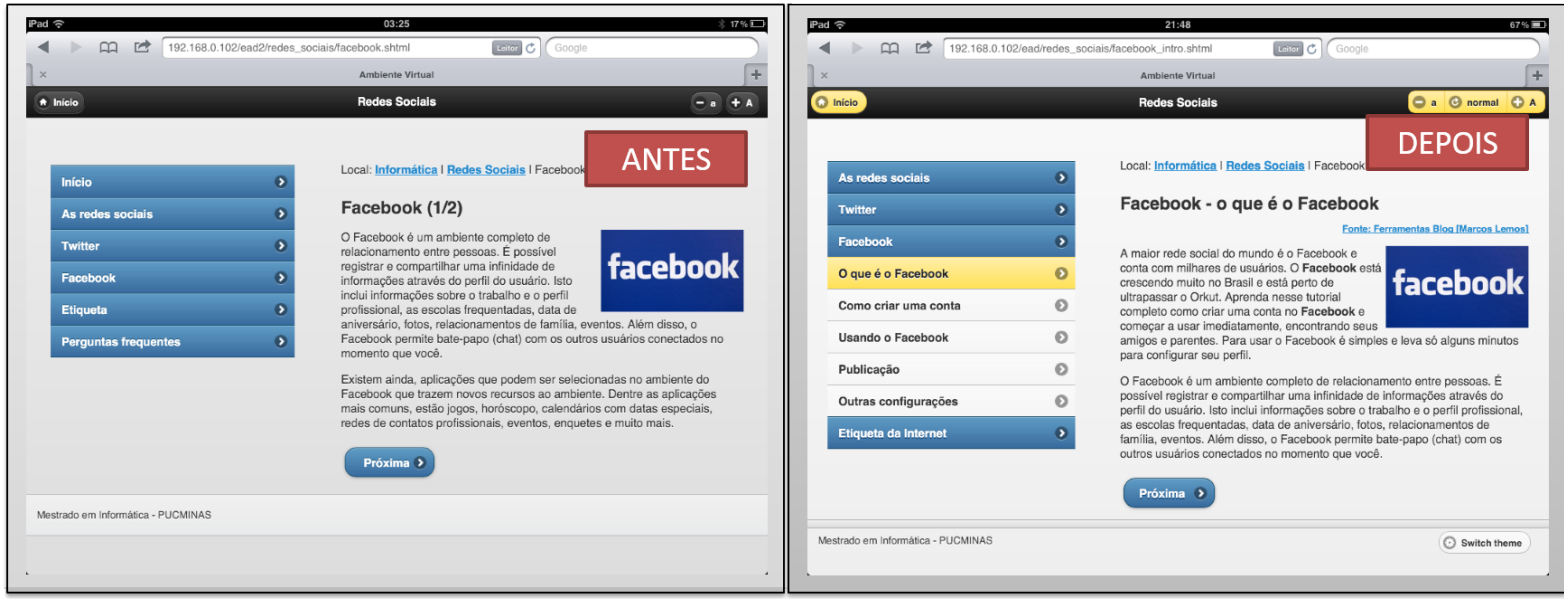

Figura 5: Navegação no smartphone - antes e depois

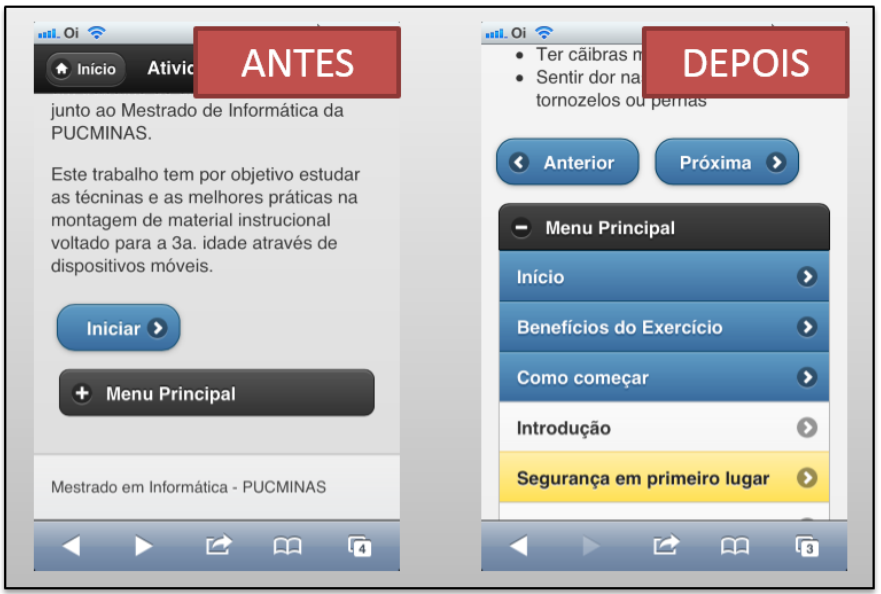

- A navegação pelo conteúdo foi identificada como um dos pontos críticos, inicialmente pela falta de indicação da localização atual no menu de opções e, posteriormente, pela confusão provocada com a navegação no 
smartphone. Este último aspecto gerou a necessidade de intervenção para orientações adicionais sobre o funcionamento do smartphone, uma vez que, em função da largura de tela reduzida, as opções de navegação transferiam-se para debaixo do conteúdo e mantinham-se fechadas até que o usuário solicitasse a abertura.

Ação de adequação: O material foi alterado para indicar de maneira mais clara o ponto onde o usuário encontrava-se (Figura 5). No caso dos smartphones, as opções de navegação que apareciam fechadas foram alteradas para estarem previamente abertas em todas as telas (Figura 5).

- Foi constatado maior interesse nos conteúdos multimídia frente ao material na forma de texto puro, tanto na comparação do tempo gasto com cada tipo de conteúdo quanto por meio de considerações feitas pelos participantes ao final do pré-teste.

Ação de adequação: Foram incluídos imagens e vídeos adicionais relacionados ao conteúdo já existente.

- Os símbolos gráficos utilizados em sistemas tradicionais não foram percebidos de maneira clara pelos usuários, requerendo uma orientação específica, a exemplo do sinal de adição (+) para expandir estruturas agrupadas e dos ícones indicativos para iniciar os vídeos $(>)$.

Ação de adequação: Foram inseridas caixas de orientação para os elementos que se valiam de símbolos gráficos (Figura 5).

Figura 6: Orientação sobre elementos de interface

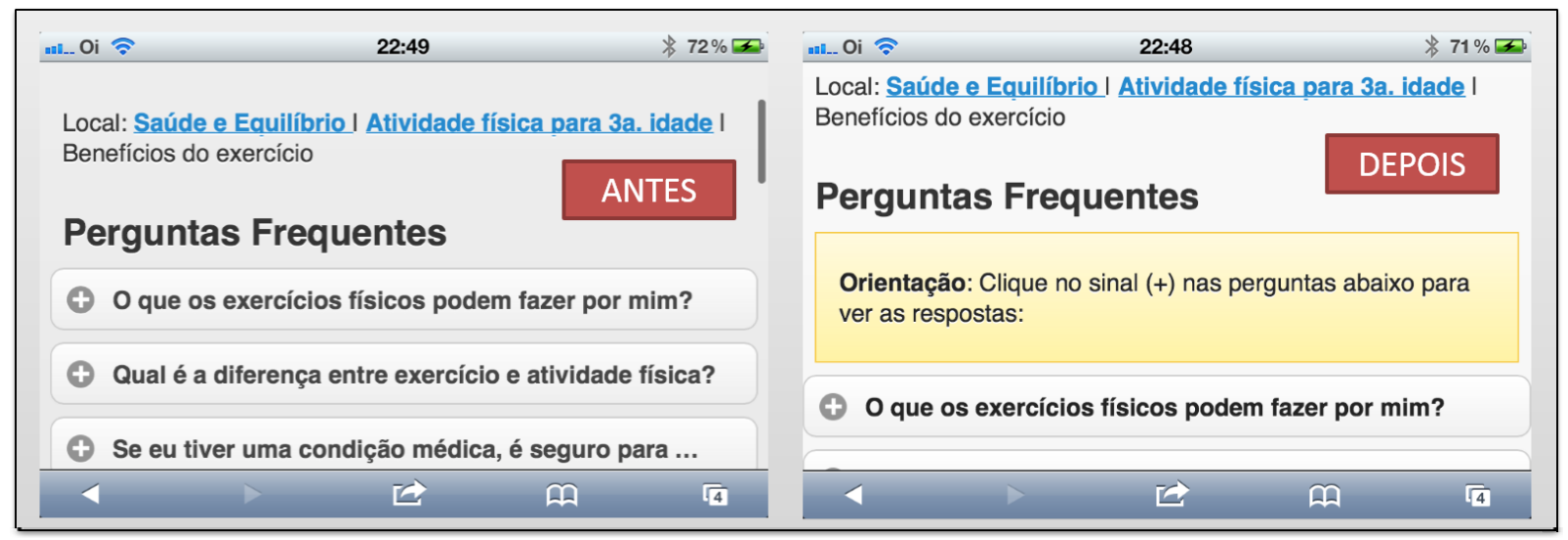

\section{Resultados do teste de usabilidade}

Os resultados apresentados nesta seção resumem os aspectos relevantes identificados durante os testes, na aplicação de dispositivos móveis nas iniciativas de aprendizagem com pessoas da terceira idade.

Os testes de usabilidade tiveram três partes, conforme descrito na Seção 4.2: um questionário, a observação do uso e entrevistas sobre a experiência dos participantes. Os testes levaram em média um tempo de 30 minutos e foram conduzidos com um grupo de 11 participantes da terceira idade, distribuídos por faixas etárias, conforme mostrado na Figura 6. Para a realização do teste, foi solicitada a escolha de um dos dois cursos disponíveis: "saúde e equilíbrio" ou "redes sociais". Os participantes foram orientados quanto ao objetivo do trabalho, as características principais dos equipamentos e a estrutura básica do conteúdo. Embora pessoas com perfis de experiência distintos tenham participado dos testes (dos 11 participantes, oito não utilizavam dispositivos móveis ou utilizavam apenas as funções básicas dos equipamentos), todos os participantes tiveram uma adaptação muito rápida ao material. Com a orientação incluída no início do teste, decorrente da necessidade identificada no pré-teste, todos os usuários conseguiram utilizar o material e passar pelas diversas partes dos minicursos.

Em algumas situações, ao contrário do esperado, pessoas nas faixas de maior idade apresentaram grande destreza na manipulação dos dispositivos móveis, enquanto indivíduos de faixas de menor idade apresentaram grande resistência.

Revista Brasileira de Computação Aplicada (ISSN 2176-6649), Passo Fundo, v. 6, n. 1, p. 81-94, abr. 2014 
Figura 7: Faixa etária dos participantes do teste

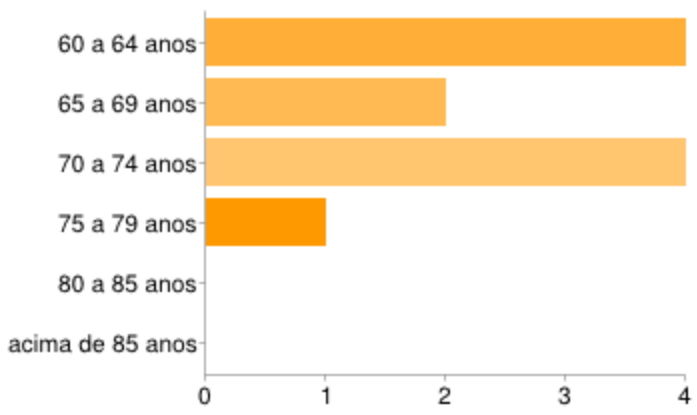

Percebe-se que existe uma carência por iniciativas que envolvam as pessoas da terceira idade com esse tipo de tecnologia, o que contribuiria muito para a vida dessa parcela da população. Isto ficou claro, uma vez que, após o teste, 9 dos 11 entrevistados manifestaram interesse em utilizar os dispositivos móveis com o objetivo de aprendizagem e informação.

As Tabelas 1 e 2 trazem extratos de algumas das falas dos usuários nas anotações feitas durante os testes com cada um dos participantes.

Tabela 1: Algumas anotações dos testes dos participantes do sexo masculino

\begin{tabular}{ll} 
Usuário/Perfil & Anotações \\
\hline \hline Usuário 03 & "Qualquer coisinha que você faz, o dedo vai no lugar errado.” \\
Idade: 72 & "Nós temos muita restrição quanto a estes dispositivos." \\
& "O software que ajusta a tela é bem interessante" \\
\hline Usuário 06 & "Eu sou mais pela praticidade." \\
Idade: 69 & "Eu uso mais o computador para e-mails, planilhas excel, digitalização, envio de docu- \\
& mentos e pagamento de contas." \\
& "Estes negócios de Twitter e Facebook não me interessa não." \\
& "Esse aqui (smartphone) é melhor que você leva pra todo lugar." \\
\hline Usuário 09 & "Eu jamais faço uso dos pagamentos na Internet. Tem os hackers." \\
Idade: 75 & "Quando se diz link, o que significa isto?" \\
& "Interessante isso. Eu nunca tinha usado esse ... Iphone?" \\
& "Eu estou impressionado de ver. É um estímulo para você ficar... Não tem limites o \\
\hline Usuário 11 & avanço das coisas." \\
Idade: 72 & Aocê quer, mas tem as dificuldades.” \\
\hline
\end{tabular}

Os resultados obtidos neste trabalho apontam, ainda, diversos aspectos de usabilidade a serem observados na condução de iniciativas de mobile learning com pessoas da terceira idade. Esses aspectos são descritos a seguir:

Dar consistência à estrutura do conteúdo - dentre os 11 participantes, seis disseram aceitar substituir os meios tradicionais já utilizados para o propósito de aprendizagem e informação, tais como livros, revistas, televisão e rádio. Do restante, três participantes substituiriam parcialmente e apenas dois afirmaram preferir os meios habituais. Do público que aceita substituir o meio habitual, apenas um tinha idade acima dos 70 anos, todos os outros se encontravam abaixo dessa faixa.

Uma das percepções resultantes dos testes de usabilidade está na estrutura do conteúdo que se traduz em termos dos mecanismos de navegação (menus de opções, elementos de interface para sequenciamento, indicadores de localização no conteúdo, entre outros), na clareza da linguagem utilizada para apresentação das informações e nos recursos de interatividade. A necessidade de uma atenção maior a este último item, a 
Tabela 2: Algumas anotações dos testes das participantes do sexo feminino

\begin{tabular}{|c|c|}
\hline Usuário/Perfil & Anotações \\
\hline Usuário 01 & "A falta de conhecimento me distancia." \\
\hline \multirow[t]{3}{*}{ Idade: 64} & "Tenho medo de colocar vírus." \\
\hline & $\begin{array}{l}\text { "O smartphone é prático por poder levar no bolso. Mas para entendimento e visibilidade } \\
\text { o tablet dá maior conforto." }\end{array}$ \\
\hline & "Eu posso comprar, mas quem me oferece este serviço?" \\
\hline Usuário 02 & "Tudo que eu quero saber, eu corro lá no computador." \\
\hline \multirow[t]{3}{*}{ Idade: 70} & "A falta de tempo impede um envolvimento maior o que dificulta o aprendizado." \\
\hline & "Tenho grande interesse em aprender a utilizar estas e outras tecnologias." \\
\hline & "Pra mim esta é uma máquina maravilhosa." \\
\hline Usuário 04 & "Já não se ouve mais a voz da pessoa. Perdendo-se o lado afetivo." \\
\hline \multirow[t]{3}{*}{ Idade: 68} & "É muito sensível." \\
\hline & "O sistema caiu e não se faz mais nada." \\
\hline & "O menino não sabia o número do telefone de casa, depois que esqueceu o celular." \\
\hline Usuário 05 & "Tenho medo de hacker. Não uso o banco pelo computador nunca." \\
\hline \multirow[t]{7}{*}{ Idade: 62} & "Eu tinha preconceito, mas hoje não.” \\
\hline & "Sabe quando você não tem muito interesse, mas sabe que vai precisar da coisa." \\
\hline & "O livro não [trocaria]. É mais fácil e agradável para folhear. Tenho prazer em manusear." \\
\hline & "Telefone pra mim é pra dar recado." \\
\hline & "Essa coisa de fofoca e facebook não me interessa. Por conta da exposição." \\
\hline & "Antigamente, a gente não sentia falta dessas coisas." \\
\hline & $\begin{array}{l}\text { "Não preciso de um celular desse porte mas vejo como algo imprescindível hoje. Um } \\
\text { negócio desse é muito prático." }\end{array}$ \\
\hline Usuário 10 & "Nunca mexi em um negócio desse (tablet)." \\
\hline Idade: 73 & $\begin{array}{l}\text { "Nos falta tempo. Isto (de utilizarmos um equipamento como este) vai acontecer pela } \\
\text { necessidade." }\end{array}$ \\
\hline
\end{tabular}

interatividade, ficou evidente durante alguns testes: primeiro quando alguns participantes começaram a se exercitar com base no curso de atividades físicas e depois na tentativa de outros já se cadastrarem nas redes sociais ao serem defrontados com as instruções do curso de redes sociais.

Utilizar controles próprios e orientação sobre a navegação - os controles nativos dos programas de navegação na Web utilizados para acesso ao conteúdo do teste de usabilidade (Figura 6) representaram um dos principais problemas durante os testes. Uma vez que o material elaborado continha todos os elementos necessários para navegação e acesso às diversas seções do conteúdo, os controles do navegador web acabavam por gerar confusão quando eram acionados acidentalmente. Dessa experiência, concluiu-se que utilizar apenas os controles do conteúdo poderiam simplificar a utilização do material.

Uma suposição errônea com relação à navegação foi identificada na utilização de símbolos e ícones indicativos de ações do sistema, comuns em sistemas de computadores, tais como abertura de menus e execução de vídeos. Após o pré-teste, constatou-se junto aos participantes que esses componentes visuais não são naturais para as pessoas que nunca tiveram contato com esse tipo de recurso. Com a inclusão de orientações explícitas sobre o funcionamento dos símbolos e ícones, os usuários conseguiram perceber a forma de utilização dos símbolos e ícones empregados e prosseguiram sem dúvidas ou questionamentos. Nas entrevistas realizadas após o teste de usabilidade, ao serem perguntados se já conheciam o significado dos símbolos utilizados, os participantes informaram que não e que as orientações incluídas os fizeram entender o funcionamento associado a esses símbolos.

Tamanho da tela faz diferença - a amplitude da tela dos tablets e consequentemente o conforto gerado pela menor frequência com que se precisa deslocar o conteúdo pela tela, levaram oito dos 11 entrevistados a optarem pelo tablet como dispositivo móvel preferido. Entre os participantes com faixa de idade acima dos 70 anos, todos optaram pelo tablet, o que sugere que o conforto na leitura, proporcionado pelo tamanho da tela, é um 
Figura 8: Controle de navegação dos dispositivos

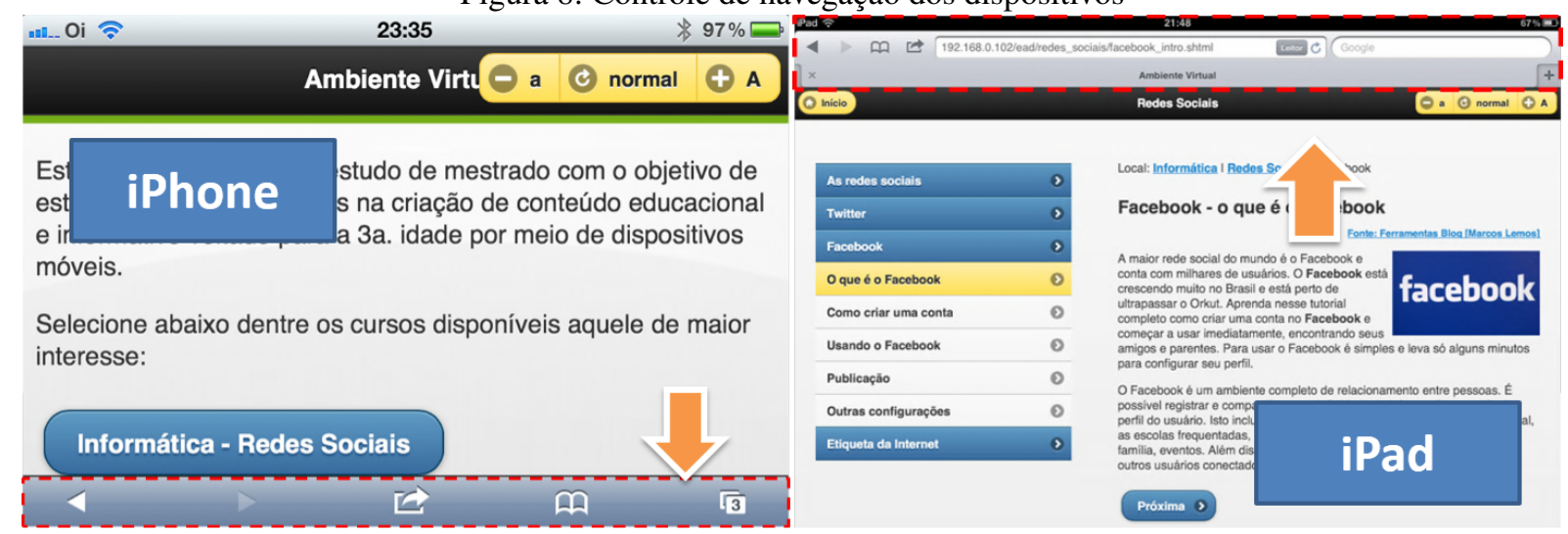

aspecto relevante para pessoas com uma idade mais avançada. Por outro lado, dois dos entrevistados, todos do sexo masculino, preferiram os smartphones. Os participantes que optaram pelo smartphone ressaltaram o benefício da mobilidade de se ter o dispositivo móvel em mais situações do dia a dia, como é o caso do Usuário 06, que deu o seguinte testemunho: "Esse aqui é melhor, pois você leva pra todo lugar".

A experiência com os dispositivos móveis baseados em tela sensíveis ao toque mostrou-se muito adequada para a maioria dos entrevistados (10 em 11), principalmente no caso dos tablets que, pela amplitude maior de tela, facilitam o controle da navegação pelo conteúdo. Embora a sensibilidade da tela dos equipamentos atrapalhasse em um primeiro contato, após alguns minutos experimentando os dispositivos utilizados no teste, os usuários mostraram-se bem adaptados a seu uso.

\section{Conclusões}

Este trabalho mostrou a aplicabilidade dos dispositivos móveis nas iniciativas de mobile learning com o público da terceira idade e fez uma avaliação dos aspectos de usabilidade relacionados. Foi possível identificar fatores que dificultam a adoção desses dispositivos por parte dos indivíduos com idade acima de 60 anos, entretanto, ficou claro que as questões encontradas podem ser contornadas com a aplicação de boas práticas na elaboração do conteúdo e com a participação de idosos na identificação das melhorias a serem aplicadas no ambiente como um todo.

O público que participou da pesquisa possui características muito divergentes entre si, porém, apesar das diferenças de perfis, foi manifestado interesse por 9 dos 11 participantes na utilização dos dispositivos para o propósito de aprendizagem e informação.

À medida que se tornam mais acessíveis e consequentemente mais difundidos, os dispositivos móveis podem atuar como meios de oferta de educação e ainda como ferramentas de interação em diversos âmbitos. Tais equipamentos podem ser utilizados como importantes agentes na promoção da inclusão digital e social. $\mathrm{O}$ trabalho de adequação dos conteúdos educacionais e informativos podem tornar essa tecnologia acessível aos idosos de maneira definitiva.

Com todas as alternativas de dispositivos móveis e seus recursos, o contexto de mobile learning mostrou-se muito dinâmico, oferecendo oportunidades variadas em termos de educação e informação. Com isso, é possível identificar algumas frentes de estudo para a continuidade deste trabalho. Conforme destacado nos estudos mais recentes sobre o assunto, a mobilidade representa uma das principais características no contexto de utilização de dispositivos móveis. Pela complexidade inicial em se realizar os testes de usabilidade de forma itinerante, e adicionalmente pela característica do próprio público da terceira idade, os testes foram realizados em cenários que não se valiam fortemente da mobilidade dos dispositivos utilizados. Um aspecto a ser explorado em novos estudos nessa área está na aplicação dos dispositivos móveis em situações de deslocamento ou durante atividades específicas de interesse do público, como atividades de lazer e ocupacionais, ou em situações ligadas a uma atividade 
profissional.

Outra possibilidade na continuidade deste trabalho está na utilização mais intensa de conteúdo interativo, o qual representa um diferencial na adoção dos dispositivos móveis ao conquistar o interesse das pessoas, não apenas da terceira idade. Uma possível abordagem futura está na inclusão de conteúdo que possa ser um guia de aplicação prática do conhecimento. A título de exemplo, alguns dos participantes do teste de usabilidade, ao passar pelo material que trazia informações sobre a utilização das redes sociais, manifestaram o interesse de interagir diretamente com essas redes como desdobramento do aprendizado.

Faz-se também necessário explorar a interação entre usuários de um ambiente voltado para a educação. Em função do conjunto de funcionalidades providas pelos dispositivos móveis, as oportunidades de interação entre os usuários de um ambiente voltado para a educação são inúmeras, como por exemplo: o correio eletrônico (email), conectividade via padrão bluetooth, recursos de áudio e videoconferências, mensagens de texto (SMS) e mensagens multimídia, localização geográfica e acesso a ambientes de redes sociais na internet. A exploração dessa gama de funcionalidades adaptadas ao ambiente de pessoas da terceira idade pode facilitar o processo de adoção de tecnologias por parte desse público e se configura como um campo rico para a pesquisa.

Por fim, diante da diversidade de tipos de dispositivos móveis, cabe também um estudo detalhado das características que essa gama de equipamentos apresenta para identificar aqueles dispositivos que são mais adequados no contexto da educação de forma geral.

\section{Agradecimentos}

Os autores agradecem à FAPEMIG (Projeto CEX-APQ-01796-09) pelo suporte financeiro.

\section{Referências}

[1] IBGE. Síntese de indicadores sociais. 2010. Disponível em: <http://www.censo2010.ibge.gov.br/resultados_ do_censo2010.php>. Acesso em: 10 jun. 2012.

[2] NAISMITH, L. et al. Literature review in mobile technologies and learning. Bristol: FutureLab, v. 11, 2004. Disponível em: <http://www.futurelab.org.uk/resources/documents/lit_reviews/Mobile_Review.pdf>. Acesso em: 14 mai. 2013.

[3] KOOLE, M. A model for framing mobile learning. In: M. Ally (Ed.), Mobile learning: Transforming the delivery of education and training. Edmonton, AB: AU Press, 2009. p. 25-47.

[4] TRAXLER, J. Learning in a mobile age. International Journal of Mobile and Blended Learning, v. 1, n. 1, p. $1-12,2009$.

[5] O'MALLEY, C. et al. Guidelines for learning/teaching/tutoring in a mobile environment. MOBIlearn deliverable $D$, v. 4, 2003.

[6] KOOLE, M.; MCQUILKIN, L.; ALLY, M. Mobile learning in distance education: utility or futility. Journal of Distance Education, v. 24, n. 2, p. 59-82, 2010.

[7] MACHADO, L. R. et al. Pedagogia, andragogia e gerontogogia: utilizando objetos de aprendizagem ao longo da vida. Práticas em Informática na Educação, v. 1, n. 1, 2010.

[8] MERRIAM, S. B.; CAFFARELLA, R. S.; BAUMGARTNER, L. M. Learning in adulthood: a comprehensive guide. Jossey-Bass, 2006.

[9] CAPRANI, N; O'CONNOR, N. E.; GURRIN, C. Touch screens for the older user. In: Assistive Technologies. Dr. Fernando Auat Cheein (Ed.). DOI: 10.5772/38302. Disponível em: <http://www.intechopen.com/books/ assistive-technologies/touch-screens-for-the-older-user>. Acesso em: 15 set. 2013.

[10] AMBROSI, A.; PEUGEOT, V.; PIMIENTA, D. Desafios de palavras: enfoques multiculturais sobre as sociedades da informação. 2005. Disponível em: <http://vecam.org/article612.html>. Acesso em: 15 set. 2013.

Revista Brasileira de Computação Aplicada (ISSN 2176-6649), Passo Fundo, v. 6, n. 1, p. 81-94, abr. 2014 
[11] RABIN, J.; MCCATHIENEVILE, C. Mobile web best practices 1.0 World Wide Web Consortium (W3C), v. 13, 2008. Disponível em: <http://www.w3.org/TR/mobile-bp/>. Acesso em: 15 set. 2013.

[12] CALDWELL, B. et al. Web Content Accessibility Guidelines (WCAG) 2.0. 2008. Disponível em: <http: //www.w3.org/TR/WCAG20/>. Acesso em: 15 set. 2013.

[13] ASSOCIAÇÃO BRASILEIRA DE NORMAS TÉCNICAS (ABNT). NBR 9241-11 - Requisitos ergonômicos para trabalho de escritórios com computadores: parte 11 - orientações sobre usabilidade. Rio de Janeiro: 2002.

[14] PREECE, J.; ROGERS, Y.; SHARP, H. Design de interação. Porto Alegre: Bookman, 2008.

[15] NAHM, E. et al. Usability of health Web sites for older adults: a preliminary study. Computers Informatics Nursing, v. 22, n. 6, p. 326-334, 2004

[16] LAW, E. L. C. et al. Understanding, scoping and defining user experience: a survey approach. In: ACM Proceedings of the 27th international conference on Human factors in computing systems. 2009. p. 719-728

[17] ARCH, A. Web accessibility for older users: a literature review. 2008. Disponível em: <http://www.w3.org/ TR/wai-age-literature/>. Acesso em: 15 mai. 2013.

[18] KURNIAWAN, S.; ZAPHIRIS, P. Research-derived web design guidelines for older people. In: Proceedings of the 7th international ACM SIGACCESS conference on Computers and accessibility. New York, NY, USA: ACM, 2005. (Assets'05), p. 129-135.

[19] National Institute of Aging - NIA. Making Your Web Site Senior Friendly - A Checklist. Disponível em: <http://www.nih.gov/icd/od/ocpl/resources/wag/documents/checklist.pdf>. Acesso em: 15 mai. 2013.

[20] PATSOULE, E.; KOUTSABASIS, P. Redesigning web sites for older adults. In: Proceedings of the 5th International Conference on PErvasive Technologies Related to Assistive Environments (PETRA'12). New York, NY, USA: ACM, 2012. DOI=hrefhttp://dx.doi.org/10.1145/2413097.241311410.1145/2413097.2413114

[21] LEITÃO, R.; SILVA, P. Target and spacing sizes for smartphone user interfaces for older aldults: design patterns based on an evaluation with users. In: Proceedings of the 19th Conference on Pattern Languages of Programs (PLoP 2012). 2012.

[22] BECKER, S. A. A study of web usability for older adults seeking online health resources. ACM Trans. Comput.-Hum. Interact., ACM, New York, NY, USA, v. 11, n. 4, p. 387-406, 2004. ISSN 1073-0516.

[23] GONÇALVES, V. P.; NERIS, V. P. d. A.; UEYAMA, J. Interação de idosos com celulares: flexibilidade para atender a diversidade. In: Proceedings of the 10th Brazilian Symposium on on Human Factors in Computing Systems and the 5th Latin American Conference on Human-Computer Interaction. Porto Alegre, Brazil, Brazil: Brazilian Computer Society, 2011. (IHC+CLIHC'11), p. 343-352.

[24] MOL, A. M.; ISHITANI, L. Avaliação de interface de um aplicativo para uso em telefone celular e voltado para a terceira idade. In: Proceedings of the IX Symposium on Human Factors in Computing Systems. Porto Alegre, Brazil: Brazilian Computer Society, 2010. (IHC'10), p. 1-10.

[25] STRENGERS, J. Smartphone interface design requirements for seniors. 2012. Dissertação (Thesis Master Information Studies).Universiteit van Amsterdam. Disponível em: <http://dare.uva.nl/document/460020>. Acesso em: 15 set. 2013.

[26] BEVAN, N. et al. The magic number 5: is it enough for web testing? In: Proceedings of the ACM CHI'03 extended abstracts on Human factors in computing systems. Fort Lauderdale, Florida: ACM, 2003. p. 698699.

[27] FAULKNER, L. Beyond the five-user assumption: benefits of increased sample sizes in usability testing. Behavior Research Methods, Springer, v. 35, n. 3, p. 379-383, 2003. 